\title{
Aportación científica de los congresos de la AE-IC (2008-2014): reseña histórica y estado actual de la investigación en Comunicación
}

\author{
Eduardo Fco. Rodríguez Gómez \\ Universidad Carlos III de Madrid \\ edrodrig@hum.uc3m.es
}

Recibido: 12 de noviembre de 2015

Aceptado: 16 de febrero de 2016

\begin{abstract}
Resumen
Esta investigación analiza la producción científica presentada en los congresos de la Asociación Española de Investigación de la Comunicación con el fin de comprender su desarrollo y su estado actual. Para ello, se han analizado 715 comunicaciones con veintidós variables, entre las que se encuentran: el autor, el género, la vinculación institucional, la nacionalidad, el tipo de comunicación, la metodología empleada, la réplica de contenidos, la tipología descriptiva y la finalidad de las comunicaciones. Entre otros hallazgos, los resultados muestran una mayoría femenina en la contribución académica, pero no entre los cargos académicos de mayor rango; la preferencia de los objetos de estudio empresariales, pero no profesionales o laborales; una tendencia hacia la mayor participación de doctorandos; y una elevada concentración de la producción científica en cuanto a la temática y la distribución territorial.
\end{abstract}

Palabras clave: AEIC; investigación en comunicación; congresos; producción científica; comunicaciones

\section{Scientific Contribution of AE-IC's Conferences (2008-2014): Historic Overview and the Current State of Communication Research}

\begin{abstract}
This paper presents a study of the Spanish scientific contribution presented at the Asociación Española de Investigación de la Comunicación conferences in order to understand its development and current state. To achieve this, twenty-two variables have been recorded for 715 papers, including: author, gender, institutional affiliation, nationality, type of communication, methodology, descriptive typology, contents replication and author's aims. Among other findings, our results show a female majority in the academic contribution, but not among senior academic positions; a preference for business studies, but not for social or working conditions studies; a trend towards a greater participation by doctoral candidates; and a high concentration of scientific production regarding territorial and thematic features.
\end{abstract}

Keywords: AEIC; communication research; conferences; scientific production; papers

\section{Referencia normalizada}

Rodríguez Gómez, E. F. (2016). Aportación científica de los congresos de la AE-IC (2008-2014): reseña histórica y estado actual de la investigación en comunicación. Historia y Comunicación Social. Vol 21, número 1, páginas 115-137. 
Sumario: 1. Introducción y estado de la cuestión. 1.1. La meta-investigación sobre comunicación. 2. Objetivos, finalidad y métodos. 3. Resultados. 3.1. Aportación general. 3.2. Género, autoría y vinculación institucional. 3.3. Universidades, proyectos y territorio. 3.4. Métodos. 3.5. Áreas de aplicación. 4. Conclusiones. 4.1. Objetivos y finalidad de las comunicaciones. 4.2. Género, autoría y vinculación institucional. 4.3. Universidades, proyectos y territorialidad. 4.4. Métodos. 4.5. Aportación y omisiones temáticas. 4.6. Limitaciones del estudio y futuras líneas de investigación. 5. Referencias bibliográficas.

\section{Introducción y estado de la cuestión}

La investigación sobre comunicación en España resulta inexistente hasta 1960, cuando se inicia la divulgación de los primeros trabajos especializados en los circuitos científicos oficiales (v.g. Mass communications de Juan Beneyto). Pero no sería hasta el final de la dictadura franquista cuando se permite la descentralización del sistema universitario (Castillo y Ruiz, 2011) y se asiste al incremento exponencial de las facultades como nodos de producción y de las publicaciones y congresos científicos como canales de divulgación. Hoy en día, 82 instituciones académicas ofertan títulos de grado o posgrado en ciencias de la información (Piñeiro-Otero y Martínez-Rolán, 2015) y existen 24 revistas científicas especializadas en la materia (Castillo, Almansa y Álvarez-Nobell, 2013; Castillo, 2011).

El surgimiento del análisis sobre la calidad de la investigación en comunicación como un nicho propio de estudio solo comienza a consolidarse a finales de la primera década del siglo XXI (vid., Moragas, 2005; Martínez-Nicolás, 2007 y 2006; Almirón, 2007; y Humanes, 2007), cuando la producción se vincula a la subvención pública y "aumentan los centros especializados en comunicación que obtienen proyectos financiados, pasando de 8 en 2004 a 17 en 2006" (Civil y Reguero, 2008).

En un principio, esta rama de la investigación estuvo ligada principalmente a la cuantificación bibliométrica del nivel de producción científica. Un registro que se realizaba mediante el conteo del número de revistas, su afiliación a los índices de medición de impacto y la internacionalización y el nivel de cooperación entre investigadores -uno de los indicadores que, según varios autores (Carrasco y Saperas-Lapiedra, 2014; De-Filippo, 2013; De Pablos, 2010), resultaba óptimo para cuantificar la apertura hacia la comunidad científica foránea y la aceptación y aplicación de sus estándares-. En función de estas variables, Fernández-Quijada y Masip (2013), Escribà y Cortiñas (2013), López-Ornelas (2011), y Alsina y García (2008) perfilaron el prototipo del investigador español: un autor de género masculino que firmaba desde una universidad española, publicaba generalmente en solitario, cuando lo hacía en grupo trabajaba con autores de la misma universidad y, en caso de colaboración internacional, las asociaciones con Latinoamérica eran las más abundantes, dado que el español era el idioma dominante. Respecto al género, el estudio coordinado por Sánchez de Madariaga (2013) revelaba que si bien las mujeres habían alcanzado la paridad en las categorías profesionales del profesorado ayudante, ayudante doctor y 
contratado doctor (49\%), su presencia era mucho menor en las categorías del profesorado titular (39\%) y muy reducida en las cátedras (19\%).

Junto a estos hallazgos, otros estudios han evidenciado una tendencia a la centralización de la investigación académica en torno a las revistas, congresos y universidades que aportan un mayor beneficio al currículum personal de los investigadores. Fernández-Quijada, Masip y Bergillos (2013), Castillo y Carretón (2010) y Giménez y Alcain (2006) indicaron como la alta concentración de la producción científica había menoscabado el lógico desarrollo de su divulgación y había consolidado la necesidad de examinar con detenimiento el estado cualitativo -no solo el cuantitativode la investigación en comunicación. Una necesidad a la que el presente artículo trata de sumarse.

\subsection{La meta-investigación sobre comunicación}

Hay dos problemas elementales que condicionan a la investigación en comunicación en España: las exigencias coyunturales de acreditación que apremian a los académicos a mostrar su productividad a través del mayor número de canales -en particular revistas científicas indexadas en bases de datos internacionales pertenecientes a grandes multinacionales, como Thomson Reuters-; y las drásticas reducciones presupuestarias que se siguen acometiendo hoy en día desde todos los estamentos, el estatal y los autonómicos, provocando el descuido de la calidad narrativa, la rigurosidad científica y el interés social o académico de las investigaciones.

La Asociación Española de Investigación de la Comunicación (AE-IC) lleva algunos años enfrentándose a estos problemas, tratando de institucionalizar la revisión de esta disciplina mediante la organización de congresos nacionales, la creación de la Comisión de Política Científica y, más recientemente, la elaboración de "un mapa que sirva de referencia para evaluar solicitudes e informes en las convocatorias de proyectos e incentivar las redes de investigación" -el proyecto, titulado MapCom (www.mapcom.es), presentará el Libro Blanco de la Comunicación en el V Congreso de la AE-IC en Madrid 2016-.

$\mathrm{Su}$ antecedente directo fue el proyecto llevado a cabo por el grupo $\mathrm{MDCS}^{1}$, de la Universidad Complutense de Madrid, que procuró conocer las características más relevantes de la investigación básica y aplicada realizada en comunicación (Piñuel, Lozano y García, 2011). Respecto a congresos y conferencias científicas, los autores apreciaron la siguiente suerte de deficiencias que otras investigaciones de referencia encontrarían transversalmente a lo largo de una década. Por un lado, el reducido número de congresos especializados frente a los macro-congresos que acaparan la mayor parte de la circulación científica y un sistema de selección que se efectúa sólo a partir de los resúmenes de las comunicaciones, calificando en muchas ocasiones la cantidad por encima de la calidad. Por otro, la presentación de trabajos con un injustificado elevado número de autores y la asistencia predominante de investigadores jóvenes y de alumnos frente a investigadores consolidados. "En el trasfondo de algunos de los problemas mencionados", concluían, "se encuentran las dificultades 
de financiación de los congresos, que impiden poner en práctica una programación más adecuada y un aumento del nivel en la selección de los asistentes y las comunicaciones" (ibíd.: 18).

Perceval y Fornieles (2008), Soriano (2008), Martínez-Nicolás (2009 y 2006), Masip (2011) y, más recientemente, Goyanes (2015), han criticado duramente el sistema científico español de la comunicación, achacando a las agencias de acreditación, especialmente la $\mathrm{ANECA}^{2}$, y a la falta de integridad de algunos investigadores, la creación de un "mandarinato" que practicaba una extraordinaria endogamia académica, fruto de la "profunda e institucionalizada burocratización de la producción académica que sufre los designios de la hiper-especialización, la ingeniería estilística y el reduccionismo estadístico". Entre otros de los citados anteriormente, Túñez y de Pablos (2013) alertaron de la invalidez de los índices de impacto para evaluar la producción científica por cuanto que medían -y miden- el número de citas de un escrito y no su calidad.

Sobre esta línea de investigación, varios autores han puesto en evidencia la evaluación positiva de la pseudo-investigación frente a los artículos de riguroso carácter metodológico. Los análisis llevados a cabo por Piñeiro-Otero y Martínez-Rolán (2015), Castillo, Almansa y Álvarez-Nobell, (2013), López-Rabadán y Vicente-Mariño (2011) y Martínez-Nicolás y Saperas-Lapiedra (2011) coincidían en señalar la escasa transparencia metodológica de las investigaciones al comprobar que solo en una tercera parte de ellas era posible identificar alguna técnica de investigación, ya fuera cuantitativa, cualitativa o de ambos tipos, y que, a pesar de que las deficiencias habían ido disminuyendo a lo largo del periodo estudiado, parecían repuntar con los trabajos más recientes. Almirón y Reig (2007) advertían de la limitación de los métodos empleados tras analizar 2.223 artículos en los que solo el $1 \%$ hacía uso de la Política Económica de la Comunicación.

\section{Objetivos, finalidad y métodos}

El objetivo general de esta investigación es analizar las comunicaciones exhibidas en los cuatro congresos que la AE-IC ha organizado hasta el momento (2008-2014) con la intención de conocer el estado cualitativo de su aportación académica, científica y social, y poder orientar a la Academia ante los retos a los que la debe enfrentarse para mejorar la investigación española en comunicación.

Para ello, se analizan veintidós variables en 715 comunicaciones -un $70 \%$ del total publicadas-, cuya selección responde a la disponibilidad para ser descargadas desde la web de la asociación y a la posibilidad de identificar a los autores que las firman. El interés por la producción científica que se presenta en los congresos de la AE-IC se justifica por ser los foros especializados que reúnen la mayor producción de conocimiento científico sobre comunicación en España. 
La clasificación de algunas variables resultó un tanto más complicada ante la carencia de instrumentos prediseñados para este tipo de análisis. A pesar de que cada vez más revistas científicas incluyen tipologías para sus artículos, no hay un precepto estandarizado que homogenice tal clasificación y cada publicación, aun con afinidades en algunas acepciones, establece sus propios criterios. Por ejemplo, Estudios sobre el Mensaje Periodístico divide sus artículos en "estudios" e "investigaciones y documentos". Comunicar establece tres tipos: "investigaciones", "informes, estudios y propuestas" y "revisiones". El Profesional de la Información tiene cuatro secciones: "observatorio", "artículos", "análisis" e "informes técnicos". Y Comunicación y Sociedad ubica los trabajos bajo una misma rúbrica: "artículos"3.

Tampoco hemos hallado una clasificación homogénea durante la revisión bibliográfica de las investigaciones de carácter bibliométrico en España. Es por ello que el análisis de contenido ha requerido una ficha de codificación ad hoc que se ha configurado una vez realizada la observación empírica de los papers. Es decir, la ficha de codificación fue enriqueciéndose a medida que se avanzaba en el análisis, de forma que las variables fueron configurándose en función de las características observadas. La clasificación de la variable "aplicación científica", por ejemplo, se ha diseñado teniendo como referente las similitudes halladas en las clasificaciones arriba mencionadas y las definiciones del DRAE 4 , pero no un parámetro estandarizado que pudiera establecerse como punto de partida.

Se ha utilizado el software IBM SPSS V22 para la aplicación del contraste chi-cuadrado en las relaciones bivariables y el software Excel 2010 para la elaboración de los gráficos. Las pruebas Chi-cuadrado han resultado estadísticamente significativas y sin casos con un recuento menor a cinco -con excepción de la relación entre las variables "Universidad pública/privada" y "Aportación social de la comunicación", que mostro 6 casillas con un recuento menor que cinco, siendo el recuento mínimo esperado de 1,86-. Respecto a los congresos de la AE-IC, el error muestral con un nivel de confianza igual a $99 \%$ es de $2,79 \%$.

Las variables son:

1. Año y lugar del congreso.

2. Sección de la comunicación.

3. Título.

4. Autor/es.

5. Vinculación institucional: catedrático, profesor (que incluye las figuras de profesor titular, contratado doctor, ayudante doctor, visitante y asociado), doctorando, licenciado y sin vinculación.

6. Género.

7. Posesión del título de doctor: sí o no.

8. Presentación de tesis doctoral: ¿la comunicación es parte de una tesis doctoral? 
9. Número de autores por comunicación.

10. Porcentaje relacional: en referencia a aquellas comunicaciones que muestran una clara relación entre ellas más allá de la afinidad temática, bien escritas por el mismo o por diferentes autores. Se trata generalmente de proyectos o tesis doctorales que se presentan por partes, por ejemplo, dos o tres grupos de investigadores que presentan los resultados o los capítulos de su proyecto en diferentes comunicaciones. En sí, las comunicaciones relacionadas no tienen por qué ser de menor contribución científica, el problema reside cuando se observan variaciones mínimas y se replican contenidos que apenas aportan a lo ya expresado en una comunicación anterior. El presente trabajo se ocupa de medir el porcentaje de las comunicaciones relacionadas en su conjunto, sin distinguir el nivel de réplica de sus contenidos.

11. Universidad o centro al que pertenecen los autores.

12. Titularidad pública o privada de la universidad o centro.

13. Comunidad Autónoma de la universidad o el centro.

14. Nacionalidad de la universidad o el centro.

15. Proyectos $\mathrm{I}+\mathrm{D}+\mathrm{i}$ : ¿la comunicación es parte de un $\mathrm{I}+\mathrm{D}+\mathrm{i}$ ?

16. Entidad financiadora del proyecto.

17. Método: cuantitativo, cualitativo, ambos.

18. Técnica metodológica: análisis de contenidos, grupos de discusión, entrevistas en profundidad, etc.

19. Validación estadística: ¿la comunicación incluye cálculos estadísticos que validan empíricamente la representatividad de las técnicas utilizadas?

20. Aplicación empírica: que surge de la observación de dos parámetros: las metodologías científicas que se han adoptado y los elementos de subjetividad que se manifiestan en el texto5.

- Investigaciones: exploraciones empíricas y sistemáticas que aportan datos, conocidos o no, sobre un concepto o fenómeno haciendo uso de métodos científicos propios de las ciencias sociales. Confirman o descartan suposiciones no validadas empíricamente.

- Revisiones: revisiones empíricas y exhaustivas que aportan datos, conocidos o no, sobre un concepto o fenómeno sin usar métodos científicos propios de las ciencias sociales. Por ejemplo, la revisión de la legislación vigente, la elaboración de listados de nuevos modelos de negocio o las revisiones históricas.

- Estudios: análisis no científicos y no exhaustivos que aportan datos exploratorios, conocidos o no, sobre un concepto o fenómeno, como la descripción de un canal de comunicación o las definiciones basadas en revisiones bibliográficas. La diferencia entre una investigación o una 
revisión y un estudio es que este último contiene elementos subjetivos no extrapolables y no validados empíricamente.

- Ensayos: reflexiones personales sobre un concepto, tema o disciplina.

- Propuestas: propuestas metodológicas que han sido comprobadas empíricamente.

- Presentaciones: comunicaciones que presentan una parte de un proyecto de investigación o de una tesis doctoral, como el estado de la cuestión o su metodología, sin incluir resultados ni comprobaciones empíricas. Las comunicaciones que solo anuncian métodos en proceso de desarrollo o revisiones bibliográficas específicas no han sido consideradas como "Propuestas" sino como "Presentaciones".

21. Nivel explicativo ${ }^{6}$ : por el que se establece el nivel de explicación que las comunicaciones muestran de sus objetos de estudio.

- Comunicaciones exploratorias: aquellas que muestran resultados orientativos y no extrapolables respecto a los objetivos fijados. Todas las comunicaciones clasificadas como "estudios" son exploratorias, mientras que todas las "revisiones" son descriptivas.

- Comunicaciones descriptivas: describen los hechos como son observados pero no explican las razones que los subyacen. Por ejemplo, cuando una investigación establece que los alumnos de periodismo utilizan poco las tecnologías de la información en sus tareas académicas y se recomienda que las instituciones competentes fomenten más su uso. Una investigación explicativa daría a conocer si los alumnos utilizan poco las tecnologías de la información y por qué es así.

- Comunicaciones explicativas: describen los hechos como son y explican las razones que los subyacen.

22. Áreas de aplicación: en referencia a la aportación de una comunicación. Es decir, en función de que sus resultados o conclusiones sean susceptibles de ser aplicados a siete áreas.

- Ciudadana: cuando los objetos o fenómenos analizados afectan a la ciudadanía directamente. Por ejemplo, investigaciones sobre medios públicos y defensa de lenguas minoritarias, ciertas revisiones sobre legislación vigente, estudios sobre género, denuncia de malas prácticas mediáticas, etc.

- Académica: cuando el conocimiento resultante es susceptible de ser aplicado al área académica de la investigación. Generalmente se trata de revisiones de temas o fenómenos con un enfoque especializado. Por ejemplo, propuestas para métodos de investigación, conceptualización de términos, etc.

- Empresarial o institucional: cuando el conocimiento aportado es susceptible de ser aplicado para el beneficio económico ${ }^{7}$ o gerencial de una o varias empresas, o una o varias instituciones públicas y ciudadanas -v. $\mathrm{g}$. 
ONGs o partidos políticos-, como los análisis de modelos de negocio o las mediciones de audiencias.

- Profesional: cuando el conocimiento aportado es susceptible de ser aplicado al buen hacer de los profesionales en comunicación -periodistas, realizadores, publicistas, etc.-.

- Laboral: cuando los resultados son susceptibles de ser aplicados al área contractual o laboral de los profesionales de la comunicación.

- Docente: cuando el conocimiento es susceptible de ser aplicado a la labor docente.

Entendemos que todas las comunicaciones tienen como finalidad ulterior la aplicación de sus resultados para la mejora del bienestar de la ciudadanía. Sin embargo, cada autor presenta diferentes niveles de aplicación para sus objetivos, de forma que para poder aprehender cuál es el nivel que se pretende poner en práctica, los objetivos se han clasificado en función del alcance que proyecta la aplicación de sus resultados. Por ejemplo, en el texto "Comunicación colectiva y salud en Madrid", el autor muestra los tres objetivos que se había propuesto lograr: "la necesidad de incrementar la investigación sobre comunicación y salud [primer nivel u objetivo principal] para mejorar el uso de las herramientas comunicativas [segundo nivel] con vistas a elevar el nivel de bienestar de la población [tercer nivel]". El fin que realmente cumple el texto es una recomendación para que los investigadores en comunicación investiguen más sobre temas de salud, y es por ello que esta comunicación quedó adscrita al área "académica", a pesar de que el objetivo último del autor fuera "elevar el nivel de bienestar de la población".

Algunas comunicaciones podían adscribirse a una o varias de estas áreas simultáneamente y, en los casos de duda -más bien marginales-, la clasificación se llevó a cabo ponderando los objetivos, las hipótesis y las conclusiones. Por ejemplo, en la investigación "El consumo mediático de los jóvenes y su influencia en los procesos de sociabilidad entre migrantes y autóctonos" los autores establecen varias pautas sobre el consumo de medios por parte de los jóvenes migrantes, lo que en principio clasificaría esta comunicación como de aportación "empresarial", ya que se estarían presentado resultados que podrían mejorar el conocimiento de las empresas sobre el consumo de medios de un sector de la población. Sin embargo, su investigación se define en torno a la idea de "que el centro escolar es el principal espacio de encuentro entre jóvenes de diferentes culturas. Los directivos y el personal docente dieron claras muestras de que contribuir en esta dirección es una de sus principales preocupaciones". Es decir, la aplicación de los resultados incide directamente sobre la necesidad de sensibilizar a los estudiantes -futuros ciudadanos- respecto a la importancia de la integración entre culturas durante el periodo escolar, quedando la comunicación clasificada como "ciudadana".

Algunos objetos de estudio, como aquellos relacionados con ETA ${ }^{8}$, la interactividad o la inmigración se examinaron con mayor detalle, ya que el conocimiento sobre ellos o la mejora de algunos servicios podían servir tanto al interés empresarial, como al institucional o al académico. En estos casos, los resultados y conclusiones 
ayudaron a precisar la clasificación. Por ejemplo, en la investigación "Servicios interactivos en la TDT española" se describen "los errores, la falta de actualización y un deficiente funcionamiento de los elementos interactivos de la TDT", mostrando así los puntos a mejorar en los servicios que las empresas proporcionan. Aunque los autores manifiestan su propósito "de garantizar un mayor acceso de la población a estos servicios", no se confirma si son beneficiosos o de qué manera pueden serlo para la ciudadanía, por lo que esta comunicación se centró en la mejora de un servicio empresarial del que luego se obtendría un beneficio económico, es decir, quedó categorizada como "empresarial".

Una última remesa de investigaciones fijaron sus objetos de estudio en fenómenos tan concretos que solo podían responder al interés personal del investigador que las llevaba a cabo. Así ocurría con las investigaciones sobre el consumo de medios por parte de estudiantes de la asignatura "x" en la universidad " $y$ ", en el que los autores, como profesores, pidieron a sus alumnos que formaran parte de las encuestas durante la investigación. Las aportaciones de estas comunicaciones demuestran un alcance muy reducido en cualquiera de las áreas propuestas y, por lo tanto, se decidió no clasificarlas en esta variable, aunque sí quedaron registradas el número de encuestas que se realizaban a los propios estudiantes. Tampoco se han clasificado los "Ensayos", ya que en un gran número de los casos el carácter subjetivo de los textos impedía saber con un mínimo exigible de precisión la intención principal de los autores.

\section{Resultados}

\subsection{Aportación general}

1.078 comunicaciones están presentes en la página web de los cuatro congresos nacionales de la AE-IC: 370 en Santiago (2008); 279 en Málaga (2010); 283 en Tarragona (2012) y 156 en Bilbao (2014). De todas las comunicaciones presentes, 235 estaban disponibles en Santiago, 172 en Málaga, 166 en Tarragona y 142 en Bilbao. Podemos observar en el gráfico 1 la clasificación de las comunicaciones según las secciones a las que pertenecían. 
Gráfico 1. Comunicaciones según sección (\%)

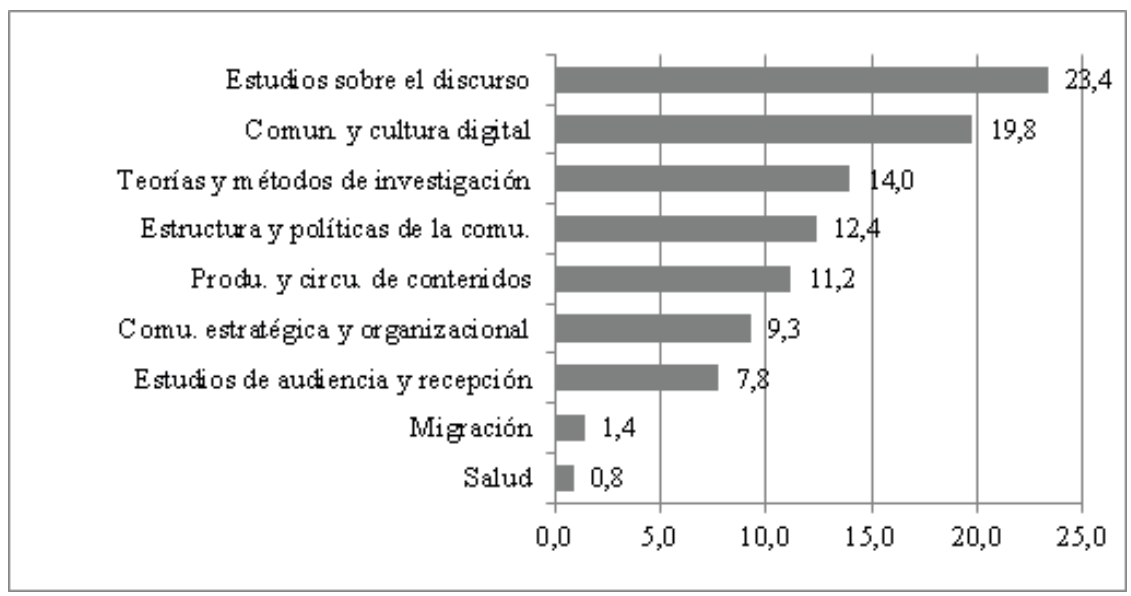

Fuente: Elaboración propia

\subsection{Género, autoría y vinculación institucional}

Históricamente, la participación femenina en la investigación siempre ha sido menor a la masculina, pero este hecho se ha invertido recientemente para las Humanidades -en torno al 2013- siendo actualmente algo superior. En nuestro análisis, un $54 \%$ de las comunicaciones fueron presentadas por mujeres y un $46 \%$ por hombres. Llama la atención que, al contabilizar el número de catedráticos y catedráticas, las mujeres resulten una minoría, solo el $21 \%$.

Según su vinculación institucional, la gran mayoría de las comunicaciones están presentadas por profesores (67\%), seguidos de los doctorandos (19\%) y los catedráticos $(3 \%)$, aunque se observa una tendencia al incremento de la participación de doctorandos al tiempo que disminuye la participación del profesorado (Gráficos 2.1. y 2.2.).

De los doctorandos que enviaron una comunicación a alguno de los congresos, el $44 \%$ presentaron su tesis, de los cuáles el $65 \%$ solo mostraron parte de ella (es decir, su comunicación era en realidad una presentación). Y en relación con el total de las comunicaciones, solo el 5\% de ellas fueron textos en los que el director/a de la tesis firmaba el artículo junto con su doctorando?

Respecto a las coautorías, el 51\% por ciento de las comunicaciones están escritas por un solo autor. Aun así, los resultados muestran una ligera tendencia a la colaboración, con porcentajes que aumentan desde el 2008 al 2014 para los trabajos de dos, tres o más autores, y que disminuyen para los trabajos de un solo autor -del $56 \%$ en el 2008 al $41 \%$ en el 2014-. 


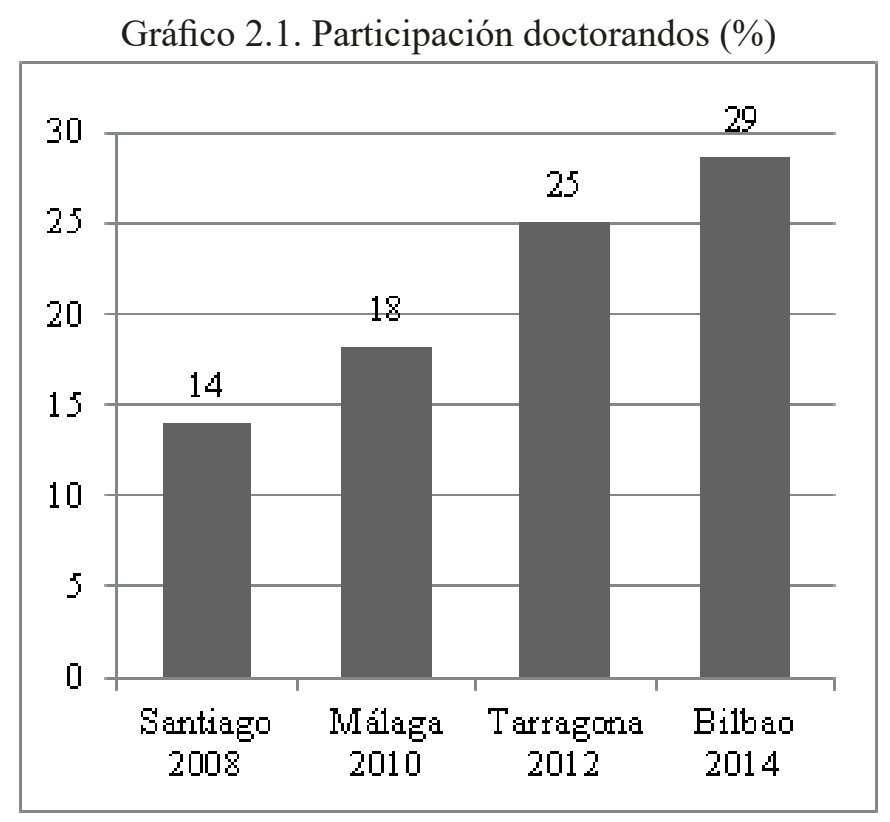

Fuente: Elaboración propia

Gráfico 2.2. Participación profesores (\%)

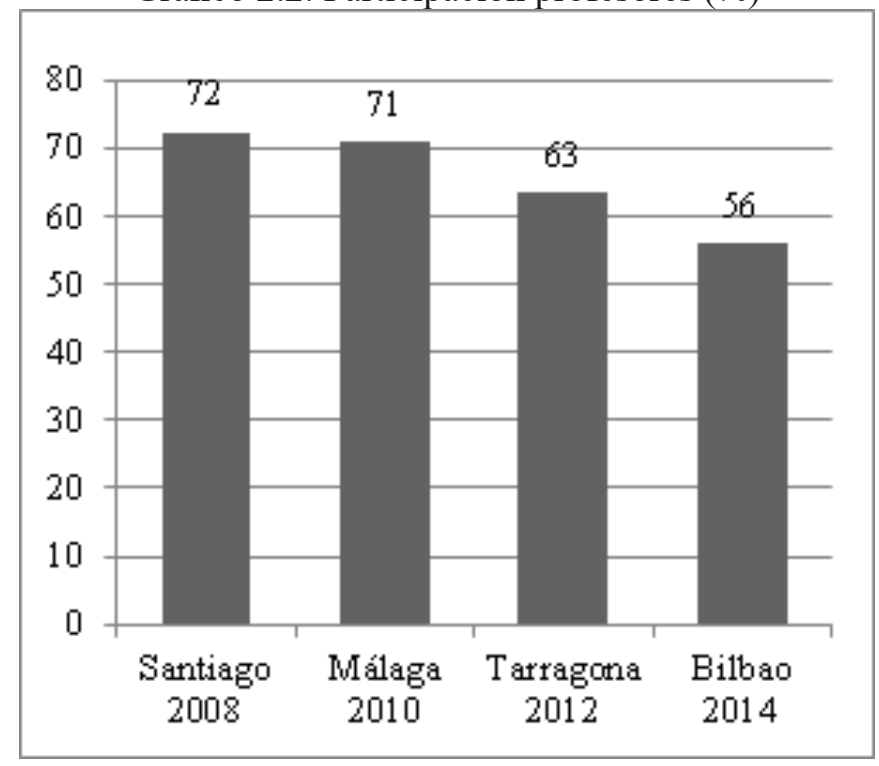

Fuente: Elaboración propia 


\subsection{Universidades, proyectos y territorio}

Estas son las diez universidades que más comunicaciones han presentado (tabla 1):

\begin{tabular}{|l|c|}
\hline \multicolumn{2}{|c|}{ Tabla 1. Número de comunicaciones según universidad } \\
\hline Euskal Herriko Unibertsitatea & 111 \\
\hline Universidad Complutense de Madrid & 105 \\
\hline Universitat Autònoma de Barcelona & 87 \\
\hline Universidad Rey Juan Carlos & 84 \\
\hline Universidad de Sevilla & 73 \\
\hline Universitat Ramón Llull & 65 \\
\hline Universidad de Salamanca & 59 \\
\hline Universidade de Santiago de Compostela & 52 \\
\hline Universidad de Valladolid & 41 \\
\hline Universitat Pompeu Fabra & 41 \\
\hline
\end{tabular}

De todas las universidades y centros universitarios que han presentado comunicaciones -un total de 137-, el 79\% eran públicas y el $21 \%$ privadas. El estudio confirma que la adjudicación de proyectos subvencionados es prácticamente igual para ambos tipos de titularidad, $9 \%$ para la privada y $11 \%$ para la pública.

Respecto al número de proyectos subvencionados, podemos observar en el gráfico 3 una tendencia a la disminución de subvenciones, aunque se requeriría de un trabajo longitudinal de mayor extensión para confirmar dicho indicio.

Gráfico 3. Número de proyectos según congreso (\%)

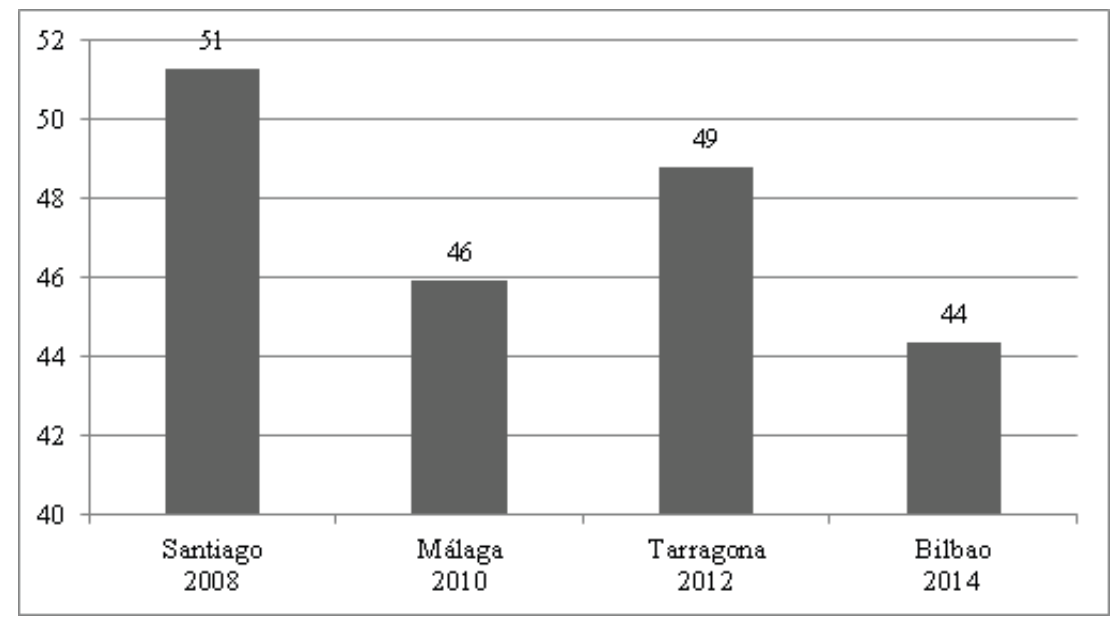

Fuente: Elaboración propia 
Resulta lógico que las Comunidades Autónomas en las que se ha celebrado alguno de los congresos muestren un mayor índice de participación (Gráfico 4), con excepción de Madrid que, al acoger el congreso del 2016, lo aumentará considerablemente.

Gráfico 4. Número de comunicaciones según Comunidad Autónoma de la Universidad

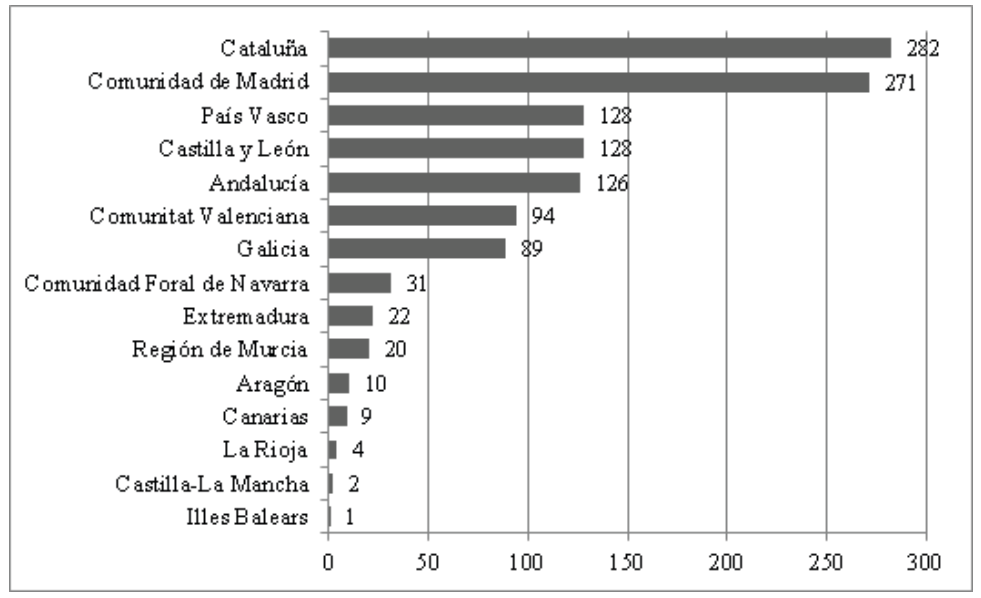

Fuente: Elaboración propia

La AE-IC promociona sus congresos como eventos internacionales, pero el gráfico 5 muestra que la gran mayoría de las comunicaciones son nacionales. Las colaboraciones fuera de nuestras fronteras se realizan principalmente con universidades latinoamericanas, lo que demuestra que el idioma español continúa siendo la lengua preferente para las relaciones internacionales.

Gráfico 5. Número de comunicaciones según nacionalidad de las universidades

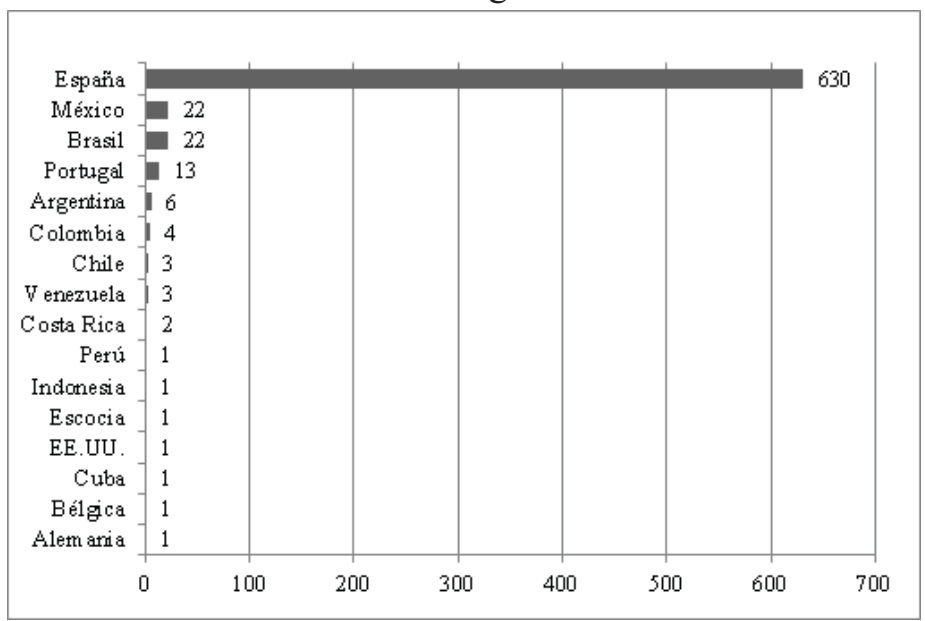

Fuente: Elaboración propia 


\subsection{Métodos}

Según la aplicación empírica, prevalecen las investigaciones, seguidas de las presentaciones y los estudios (gráfico 6). También hemos observado una tendencia al incremento en el número de investigaciones: del 15\% en 2008, al 39\% en 2014.

Gráfico 6. Tipo de comunicación según la variable "aplicación empírica” (\%)

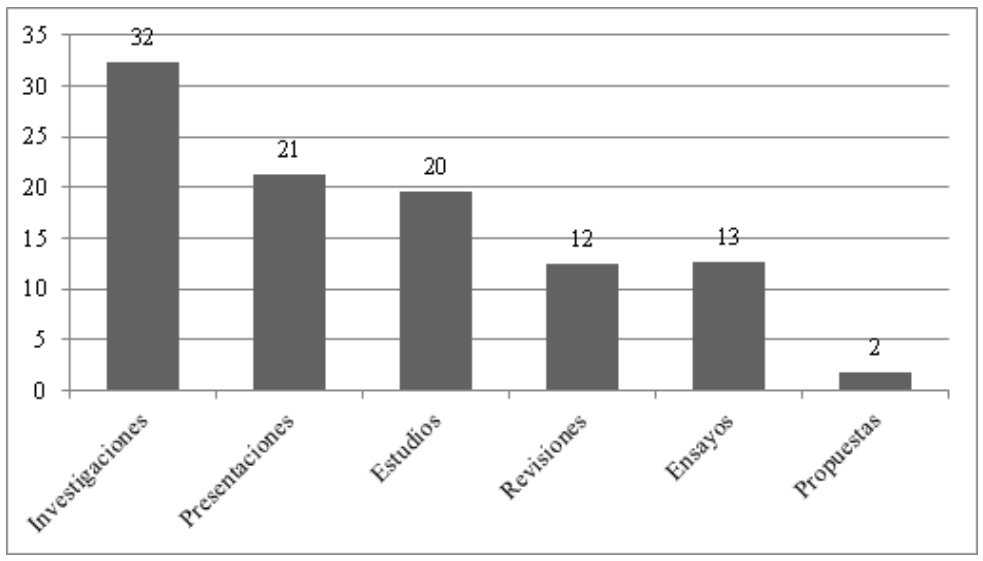

Fuente: Elaboración propia

Del total de las investigaciones, solo el $14 \%$ presentaba métodos de validación estadística. Resulta interesante observar la diferencia en la aplicación de validación estadística dependiendo de si la investigación era resultado de un proyecto subvencionado o no (gráficos 7.1. y 7.2.).

Gráfico 7.1. Validación empírica y proyectos no subvencionados (\%)

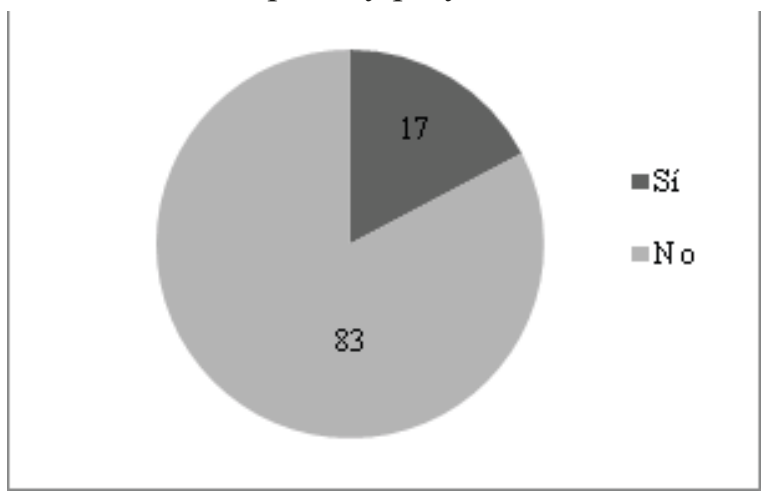

Fuente: Elaboración propia 
Gráfico 7.2. Validación empírica y proyectos subvencionados (\%)

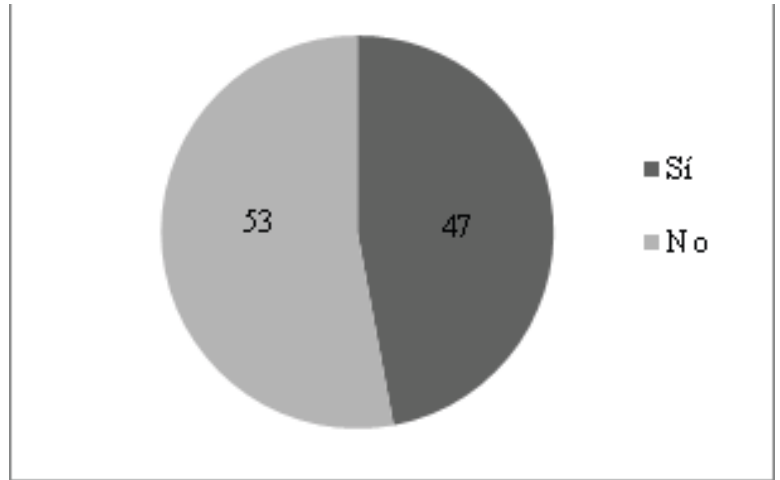

Fuente: Elaboración propia

$\mathrm{Al}$ igual que en estudios precedentes, la mayoría de las investigaciones utiliza los análisis de contenidos cuantitativos descriptivos (un 53\%), seguidos de las encuestas (21\%) y las entrevistas en profundidad (15\%). Respecto a las encuestas, el 30\% (solo 16) se realizan con estudiantes de la universidad a la que pertenecen los autores, lo que anula prácticamente la representatividad de estos sondeos salvo para los dos casos en los que los objetos de estudio eran los propios universitarios.

\section{5. Áreas de aplicación}

La mayoría de las comunicaciones son de carácter empresarial (39\%), seguidas de las comunicaciones académicas (27\%), las dirigidas a la ciudadanía (13\%), las institucionales (13\%) y, por último, las de carácter profesional (4\%), las docentes $(3 \%)$ y las de carácter laboral (1\%). En los gráficos 8.1. y 8.2 podemos ver las diferencias en las áreas de aplicación según la titularidad, pública o privada, de las universidades.

Gráfico 8.1. Aportación de las comunicaciones según titularidad pública (\%)

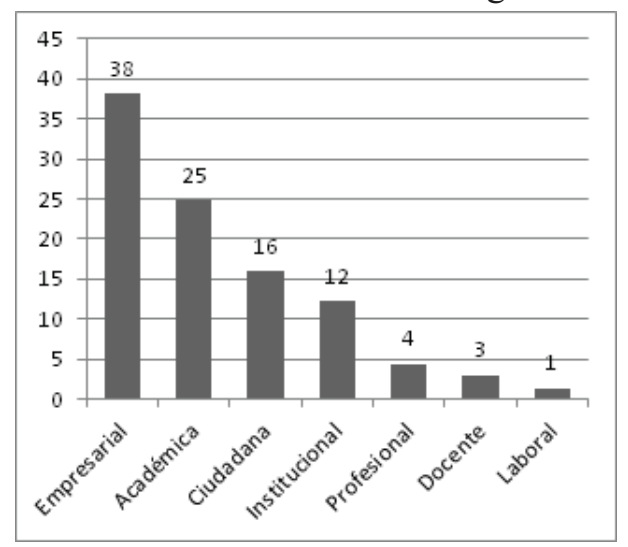

Fuente: Elaboración propia 
Gráfico 8.2. Aportación de las comunicaciones según titularidad privada (\%)

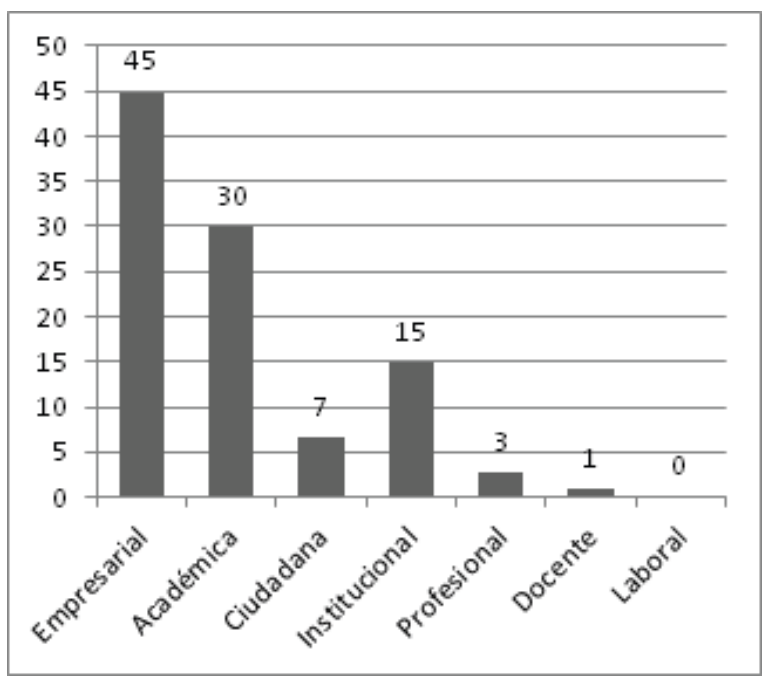

Fuente: Elaboración propia

Solo seis comunicaciones de las 715 analizadas se preocupan por las mejoras en las condiciones contractuales o laborales de los profesionales de la comunicación. Once comunicaciones hacen referencia a medios comunitarios o del tercer sector, y no hay ninguna comunicación dedicada al periodismo de investigación.

El 17\% del total de las comunicaciones muestran un factor relacional más allá de la afinidad temática, lo que muestra la intención del autor/a por replicar contenidos de un congreso a otro. Sin embargo, parece haber una tendencia a reducir esta práctica y presentar cada vez más trabajos originales (gráfico 9).

Gráfico 9. Número de comunicaciones relacionadas según congreso (\%)

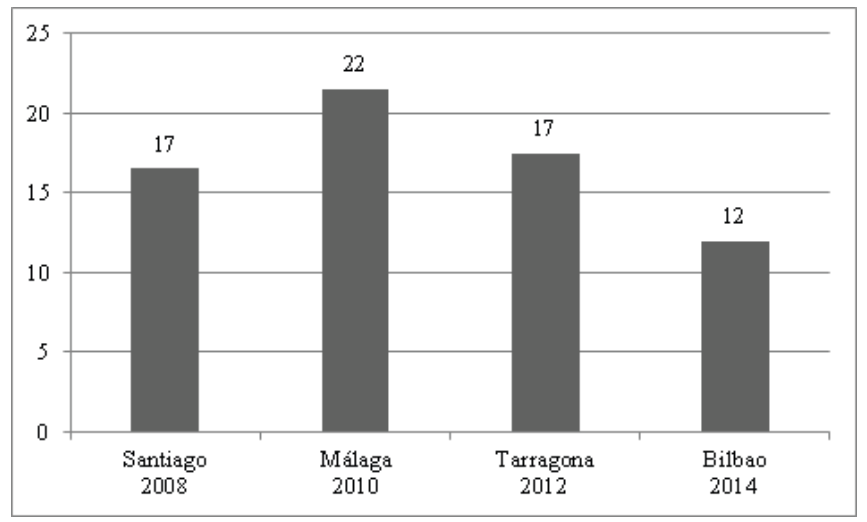

Fuente: Elaboración propia 


\section{Conclusiones}

\subsection{Objetivos y finalidad de las comunicaciones}

Por regla general, los investigadores precisan los objetivos a cumplir en función del trabajo que van a realizar y que finalmente culminan, pero no definen cuál es la finalidad de sus investigaciones, siendo una minoría los investigadores que definen sus objetivos con dos indicaciones que revelan la aportación de sus trabajos: a quiénes va dirigida su investigación y qué utilidad pudiera brindar a ese colectivo o entidad. Lo más común en la enunciación de los objetivos son fórmulas del tipo: "este artículo tiene como objetivo analizar los medios escritos de mayor circulación según la teoría del...".

De igual forma, los títulos de las "investigaciones" deberían ajustarse a los objetivos fijados, evitando la generalización en las conclusiones cuando lo que se estudia es un caso en particular -una de las razonas por las que la mayoría de las comunicaciones son exploratorias (64\%), un tercio descriptivas (34\%) y solo un $1 \%$ explicativas-. Por ejemplo, el título ficticio "La información politizada respecto a los actos violentos de grupos nacionalistas" no se corresponde con un estudio sobre las noticias publicadas en tres periódicos nacionales tras un atentado terrorista, ya que tres diarios nacionales no representan a la totalidad de la prensa en España y mucho menos a la prensa nacionalista local.

\subsection{Género, autoría y vinculación institucional}

Es posible que el desequilibrio entre el número de autoras y el número de catedráticas se deba a la paralización del sistema de reposición docente que ha bloqueado el acceso a los cargos funcionariales. Puesto que la implicación de las mujeres en la producción científica ha incrementado en los últimos años, superando en la actualidad a la de los hombres -al menos en Humanidades-, y siempre que no intercedan otras imposiciones ajenas a la tasación fundamentada del régimen evaluador, es de esperar que la recuperación de un ritmo lógico en la renovación del profesorado haga que el número de catedráticas sea más afín a la realidad del sistema docente investigador, aunque parece que llevará su tiempo.

La gran mayoría de las comunicaciones son trabajos originales presentados por el profesorado universitario, bien catedráticos, profesores titulares u otras figuras docentes, y aunque parece existir la creencia de que muchos profesores y catedráticos aparecen como autores en trabajos realizados por sus estudiantes, este tipo de colaboraciones representan tan solo un 5\% del total de las comunicaciones examinadas, lo que demuestra que, a pesar de que el fenómeno existe y que debiera erradicarse si la coautoría no es genuina, se trata de una práctica casual que en principio no desmejora el estado cualitativo de la investigación.

Por otro lado, es lógico que la mayoría de los investigadores nóveles aprovechen este tipo de congresos para compartir sus hallazgos con otros académicos. En el caso 
de la AE-IC, 179 doctorandos han participado en sus congresos, de los cuales el 29\% han presentado parte de sus tesis sin resultados. Por ello y ante el aumento del número de doctorandos, sería conveniente que los evaluadores disminuyeran al mínimo la inclusión de tesis -y proyectos de $\mathrm{I}+\mathrm{D}+\mathrm{i}-$ que no presenten resultados originales o, al menos, parte de ellos. Entendemos que un congreso internacional y no especializado en una temática o sector concreto debería servir de plataforma para la divulgación del conocimiento científico y no tanto para la presentación del desarrollo de las investigaciones de los investigadores. Quizás sea conveniente crear mesas de trabajo específicas para este propósito, en las que las tesis no concluidas, por ejemplo, puedan ser debatidas por académicos afines a su temática.

Respecto al nivel de coautoría, a pesar de una leve tendencia hacia el trabajo cooperativo, las comunicaciones en España siguen escribiéndose por un solo autor. Puede que este hecho se relacione con la disminución en las subvenciones para proyectos de $\mathrm{I}+\mathrm{D}+\mathrm{i}$, en los que se requiere el trabajo grupal. Debido a la dificultad para formar grupos de investigación, los investigadores acometen individualmente el estudio de los objetos de su interés haciendo uso de los recursos que más tienen a mano: su conocimiento, su criterio y su tiempo. Los investigadores españoles no podemos hacer mucho más al respecto, salvo intentar formar parte en los selectivos grupos de investigación o buscar financiación fuera del circuito público de adjudicaciones. Una de las acciones que podría mejorar esta situación, es el apoyo de las estancias directivas de las facultades para buscar sinergias en la formación de equipos de investigación que sean susceptibles de recibir ayudas económicas, haciendo extensible la participación a otras disciplinas como la informática, la sociología, la psicología -sobre todo para temas de aplicación al área docente-, o la semiología.

A fin de identificar con precisión el origen de las comunicaciones y poder analizar la contribución general de este u otros congresos, es recomendable que los autores se identifiquen adecuadamente en sus textos, incluyendo su nombre completo, la universidad a la que representan, su afiliación laboral y alguna forma de contacto.

\subsection{Universidades, proyectos y territorialidad}

Sigue habiendo una fuerte concentración de la divulgación científica en torno a las mayores capitales de provincia, a pesar del evidente esfuerzo inicial de los organizadores de la AE-IC para diseminarla. Es recomendable mantener el espíritu descentralizador de los tres primeros congresos con el propósito de dar visibilidad a instituciones docentes más pequeñas o a investigadores con recursos limitados para la movilidad.

En cuanto a la internacionalización de nuestras investigaciones, los autores españoles seguimos siendo predominantemente nacionales, con la gran mayoría de las cooperaciones internacionales vinculadas a instancias docentes latinoamericanas (un $95 \%$ del total de las autorías en grupo). 


\subsection{Métodos}

Resulta evidente que la calidad de las comunicaciones se ve mermada por la falta de recursos. Prueba de ello es la disminución en el número de proyectos financiados, el reducido número de investigaciones empíricas con validación estadística, el uso de la encuesta entre estudiantes universitarios, los estudios no extrapolables y las presentaciones sin resultados. Estos datos nos indicarían una tendencia hacia la falta de investigaciones susceptibles de ser replicables y comparables en diferentes contextos, a modo de canon internacional para una investigación aplicada.

Es también evidente que el número de investigaciones ha ido en aumento con el paso de los años, pero el uso extendido del análisis de contenido descriptivo con un solo autor muestra igualmente la falta de recursos económicos para usar otro tipo de métodos o instrumentos de medición más complejos o exhaustivos.

\subsection{Aportación y omisiones temáticas}

Cerca de la mitad de las comunicaciones muestran unos resultados susceptibles de ser aplicables al área empresarial y poco menos de un tercio a la académica, lo que deja muy poco espacio para la investigación que se preocupa por mejorar el conocimiento ciudadano o las condiciones laborales de los trabajadores del sector. En principio, las recomendaciones para mejorar los productos o servicios de las empresas con ánimo de lucro terminarían beneficiando a los redactores o a los consumidores finales, pero el primer nivel de alcance de las comunicaciones caracterizadas como "empresariales" poseen la intención de mejorar un producto o servicio que lleva anexa su venta y, por lo tanto, el propósito principal de obtener un beneficio mercantil. El hecho de que los consumidores puedan beneficiarse de esa mejora empresarial, da pie a un extenso debate.

Las comunicaciones adscritas a la sección "Comunicación y cultura digital" suponen el $20 \%$ del total y se aprecia, respecto al estudio de las redes sociales, cierta ausencia de un enfoque crítico para el análisis de estas empresas o fenómenos, así como de las tecnologías y repercusiones ligadas a ellas, siendo la gran mayoría de estas comunicaciones de carácter descriptivo.

\subsection{Limitaciones del estudio y futuras líneas de investigación}

Aunque los resultados son estadísticamente representativos de la investigación presentada en los congresos de AE-IC, solo son orientativos respecto al estado actual de la investigación en comunicación presentada en España. Solo un estudio posterior que analizara los artículos publicados en las revistas de investigación españolas más representativas podría ofrecer unos resultados más afines a la realidad de la disciplina, especialmente si hacemos uso de la tabla de variables diseñada para la presente investigación, permitiendo el contraste entre ambos estudios. 


\section{Referencias bibliográficas}

ALMIRÓN, Núria (2007). "la economía política de la investigación informacional". Revista latina de comunicación social, 62. http://www.ull.es/publicaciones/latina/200716almiron.htm

ALMIRON, Núria; REIG, Ramón (2007). "The Communications Research in Spain: the Political Economy. Epistemological Approach". American Communication Journal, Vol. 9, No. 2. https://idus.us.es/xmlui/handle/11441/28229

ALSINA, Rodrigo; GARCÍA, Leonarda (2008). "Teoría e investigación de la comunicación en España: Notas para un debate". En: I Congreso de Asociación Española de Investigación en Comunicación, 2008. http://www.ae-ic.org/santiago2008/ contents/pdf/comunicaciones/32.pdf

CARRASCO, Ángel; SAPERAS-LAPIEDRA, Enric (2014). "Tendencias internacionales de la investigación en comunicación actual: modalidades de investigación, objetos de estudio y usos de la teoría en los papers de Journal of communication (2008-2013)". En: I Congreso de Asociación Española de Investigación en Comunicación, 2008. www.aeic2014bilbao.org/download/aeic2014bilbao_comunicaciones.pdf

CASANUEVA, Cristóbal; CARO, Francisco Javier (2013). "La Academia Española de Comunicación: productividad científica frente a actividad social [Spanish Communication Academia: Scientific Productivity vs. Social Activity]". Comunicar, 41, pp. 61-70. DOI: http://dx.doi.org/10.3916/C41-2013-06

CASTILLO, Antonio (2011). "El rol de las publicaciones científicas en Comunicación en el EEES: indexación e impacto". Revista Internacional de Relaciones Públicas, Vol. I, 1, pp. 135-154. Última consulta: 7 de noviembre de 2015.

CASTILLO, Antonio; CARRETÓN, María del Carmen (2010). "Investigación en Comunicación. Estudio bibliométrico de las Revistas de Comunicación en España". Comunicación y sociedad, Vol. 23 (2), pp. 289-327. http://www.unav.es/ fcom/communication-society/es/resumen.php?art_id $=372$

CASTILLO, Antonio; RUIZ, Isabel (2011). "Reflexión sobre las claves de acceso y visibilidad de las revistas españolas de Comunicación". En: Fonseca-Mora, M.C. (Coord.) Acceso y visibilidad de las revistas cientificas españolas de Comunicación. Tenerife: Colección Cuadernos Artesanos de Latina, 10, pp. 8-22. http:// rabida.uhu.es/dspace/handle/10272/5321

CASTILLO, Antonio; ALMANSA, Ana; ÁLVAREZ-NOBELL, Alejandro (2013). "Metodología científica en las revistas españolas de comunicación". En: II Congreso Nacional de Metodología de la Investigación en Comunicación, 2013. http://dialnet.unirioja.es/descarga/articulo/4227307.pdf

(2012). "La Investigación en comunicación. Análisis bibliométrico de las revistas de mayor impacto del ISI". Revista Latina de Comunicación Social, 67, pp. 248-270. DOI: 10.4185/RLCS-067-955-248-270. http://www.revistalatinacs. org/067/art/955_UMA/11_Castillo.html

CIVIL, Marta; REGUERO, Núria (2008). "La investigación en comunicación en España. Análisis de los proyectos competitivos aprobados en el marco del Plan Nacional I+D+I 2004-2007”. En: I Congreso de Asociación Española de Investi- 
gación en Comunicación, 2008. http://www.ae-ic.org/santiago2008/contents/pdf/ comunicaciones/334.pdf

DE-FILIPPO, Daniela (2013). "La producción científica española en Comunicación en WOS. Las revistas indexadas en SSCI (2007-12) [Spanish Scientific Output in Communication Sciences in WOS. The Scientific Journals in SSCI (2007-12)]". Comunicar, 41, pp. 25-34. DOI: http://dx.doi.org/10.3916/C41-2013-02

DE PABLOS, José Manuel (2010). "Análisis de las revistas españolas de Comunicación, tras la actualización del índice de impacto de 2009 (octubre de 2010)". En: II Congreso International Latina de Comunicación Social, 2010. http://www. revistalatinacs.org/10SLCS/actas_2010/217_De_Pablos.html

ESCRIBÀ, Eudal; CORTIÑAS, Sergi (2013). "La internacionalización y las coautorías en las principales revistas científicas de Comunicación en España [Internationalization and Coauthorship in Major Communication Journals in Spain]". Comunicar, 41, pp. 35-44. DOI: http://dx.doi.org/10.3916/C41-2013-03

FERNÁNDEZ-QUIJADA, David; MASIP, Pere (2013). "Tres décadas de investigación española en comunicación: hacia la mayoría de edad [Three Decades of Spanish Communication Research: Towards Legal Age]”. Comunicar, 41, pp. 15-24. DOI: http://dx.doi.org/10.3916/C41-2013-01

FERNÁNDEZ-QUIJADA, David; MASIP, Pere; Bergillos, Ignacio (2013). “El precio de la internacionalidad: la dualidad en los patrones de publicación de los investigadores españoles en comunicación". Revista Española de Documentación Cientifica, 36 (2): e010. DOI: http://dx.doi.org/10.3989/redc.2013.2.936.

GIMÉNEZ, Elea; ALCAIN, María Dolores (2006). "Estudio de las revistas españolas de periodismo". Comunicación y Sociedad, XIX (2), pp. 107-131. http://www. unav.es/fcom/communication-society/es/articulo.php?art_id=62

GOYANES, Manuel (2015). “Hacia una investigación estandarizada?” Observatorio (OBS) Journal, 9, (3), pp. 85-99. http://obs.obercom.pt/index.php/obs/article/ view/826

HUMAMES, María Luisa (2007). "La investigación sobre la profesión periodística en España". Sphera pública, número especial. http://www.researchgate.net/publication/255717490_La_investigacin_sobre_la_profesin_periodstica_en_Espaa

LÓPEZ-ORNELAS, Maricela (2011). "Los procesos de comunicación de Revista Latina de Comunicación Social (RLCS)". En: Fonseca-Mora, M.C. (Coord.) Acceso y visibilidad de las revistas cientificas españolas de Comunicación. Tenerife: Colección Cuadernos Artesanos de Latina, 10, pp. 82-106. http://rabida.uhu. es/dspace/handle/10272/5321

LÓPEZ-RABADÁN, Pablo; VICENTE-MARIÑO, Miguel (2011). "Métodos y técnicas de investigación dominantes en las revistas científicas españolas sobre comunicación (2000-2009)". En: I Congreso Nacional de Metodología de la Investigación en Comunicación, 2011. http://www.revistacomunicar.com/pdf/2011-04Lopez-Vicente.pdf

MARTÍNEZ-NICOLÁS, Manuel (2009). "La investigación sobre comunicación en España. Evolución histórica y retos actuales". Revista Latina de Comunicación 
Social, 64, pp. 1-14. http://www.revistalatinacs.org/09/art/01_800_01_investigacion/Manuel_Martínez_Nicolás.html

(2007). "Agitación en el campo. Nueve ideas para la investigación sobre Comunicación Política en España”. Política y Sociedad, 44 (2), pp. 209-227. http:// revistas.ucm.es/index.php/POSO/article/view/POSO0707230209A/22305

(2006). "Masa (en situación) crítica. La investigación sobre periodismo en España: comunidad científica e intereses de conocimiento". Anàlisi, 33. http:// dialnet.unirioja.es/servlet/articulo? codigo $=2136360$

MARTÍNEZ-NICOLÁS, Manuel; SAPERAS-LAPIEDRA, Enric (2011). "La investigación sobre Comunicación en España (1998-2007). Análisis de los artículos publicados en revistas científicas". Revista Latina de Comunicación Social, 66, pp. 101-129. DOI: 10.4185/RLCS-66-2011-926-101-129. http://www.revistalatinacs.org/11/art/926 Vicalvaro/05 Nicolas.html

MASIP, Pere (2011). "Efecto Aneca: producción española en comunicación en el Social science citation index". Anuario ThinkEPI, 5, pp. 206-210. http://eprints. rclis.org/15944/

MORAGAS, Miquel de (2005). "Investigación de la comunicación y política científica en España". En: Reunión Cientifica de la Sociedad Española de Periodística, 2005.

PERCEVAL, José María; FORNIELES, Javier (2008). “Confucio contra Sócrates: la perversa relación entre la investigación y la acreditación”. Anàlisi, 36, pp. 213-224. http://www.raco.cat/index.php/Analisi/article/view/94533/119748

PIÑEIRO-OTERO, Teresa; MARTÍNEZ-ROLÁN, Xabier (2015). "De la reflexión al análisis cuantitativo. Una aproximación a la investigación radiofónica española". En: III Congreso Nacional de Metodología de la Investigación en Comunicación, 2015.

PIÑUEL, Jose Luis; LOZANO, Carlos; GARCÍA, Antonio (2011). "Investigar la Comunicación en España". En: I Congreso nacional de Metodología de la Investigación en Comunicación España, 2011. www.ae-ic.org/tarragona2012/contents/ download/InvestigarlaComunicacionenEspana_Abril2011.pdf

SÁNCHEZ DE MADARIAGA, Inés (coord.) (2013). "Científicas en cifras 2013. Estadísticas e indicadores de la (des)igualdad de género en la formación y profesión científica". Unidad de Mujeres y Ciencia del Ministerio de Economía y Competitividad. http:/www.idi.mineco.gob.es/stfls/MICINN/Ministerio/FICHEROS/UMYC/Cientificas_cifras_2013.pdf

SORIANO, Jaume (2008). "El efecto ANECA". En: I Congreso de la Asociación Española de Investigación en Comunicación, 2008. www.ae-ic.org/santiago2008/ contents/pdf/comunicaciones/286.pdf

TÚÑEZ, Miguel; DE PABLOS, José Manuel (2013). "El 'índice h' en las estrategias de visibilidad, posicionamiento y medición de impacto de artículos y revistas de investigación". En: II Congreso Nacional sobre Metodología de la Investigación en Comunicación, 2013, pp. 133-150. dialnet.unirioja.es/descarga/ articulo/4227310.pdf 


\section{Notas}

1 Mediación Dialéctica de la Comunicación Social.

2 Agencia Nacional de Evaluación de la Calidad y Acreditación.

3 El autor de la presente investigación se ha puesto en contacto con estas revistas para conocer con más detalle a qué atienden las diferentes clasificaciones, pero no ha obtenido respuesta.

4 Diccionario de la Lengua de la Real Academia Española.

5 Con ello, no se generan niveles superiores ni inferiores. La reflexión personal en torno a la definición de un concepto puede suponer un cambio de paradigma con importantes consecuencias para la sociedad. Desde el punto de vista de la divulgación epistemológica, una "Investigación" es tratada con la misma importancia que una "Ponencia".

6 Los resultados de esta variable son solo orientativos, no representativos.

7 En referencia a la mejora de las tareas comunicativas, no a la mejora de la situación contractual o laboral de los trabajadores.

8 Euskadi Ta Askatasuna.

9 Cabe mencionar en este punto el análisis de Casanueva y Caro (2013) sobre las redes sociales y su influencia positiva y significativa en la confirmación de los tribunales que juzgan las tesis doctorales en el campo de la comunicación, aunque estas redes son relativamente poco centralizadas y jerarquizadas, lo cual dejaba "este acontecimiento académico bastante abierto a la participación de muchos y no concentrado en estructura social con un núcleo central dominante". 